\title{
INVARIANT TENSORS UNDER THE REAL REPRESENTATION OF SYMPLECTIC GROUP AND THEIR APPLICATIONS
}

\author{
Tetsuzo Fukami
}

(Received October 10, 1957)

N.H. Kuiper and K. Yano [2] $]^{1)}$ determined all tensors of some kinds interesting in differential geometry, which are invariant under the proper orthogonal group of the $n$-dimensional vector space or under the group of proper orthogonal transformations fixing a unit vector. The present author [1] has recently studied tensors invariant under the real representation of unitary group. The purpose of this paper is to determine all tensors of the same types as they studied which are invariant under the real representation of symplectic group $S p(n)$.

In $\S 1$ we make preliminary considerations on the real representation of symplectic group. In $\S 2$ we state the main theorem and prove it in $\S \S 2,3,4$. In $\$ 5$ we give some applications of our main theorem to the theory of affinely connected manifolds with almost complex, quaternion or Hermitian structure, which has recently been developed by M. Obata [3].

1. Let $V$ be a $4 n$-dimensional real vector space and let there be given in $V$ a metric tensor $g_{i h}$, and tensors $\phi_{i}{ }^{h}$ and $\psi_{i}{ }^{h}$ such that

$$
\begin{aligned}
& \phi_{k}{ }^{h} \phi_{i}{ }^{k}=-\delta_{i}{ }^{h}, \psi_{k}{ }^{h} \psi_{i}{ }^{k}=-\delta_{i}{ }^{h}, \phi_{k}{ }^{h} \psi_{i}{ }^{k}=-\psi_{k}{ }^{h} \phi_{i}{ }^{k}, \\
& \phi_{i}{ }^{a} g_{a_{h}}+\phi_{h}{ }^{a} g_{a i}=0 \quad \text { and } \quad \psi_{i}{ }^{a} g_{a h}+\psi_{h}{ }^{a} g_{a i}=0 .
\end{aligned}
$$

The group composed of all linear transformations of $V$ leaving invariant the tensors $g_{i h}, \phi_{i}{ }^{h}$ and $\psi_{i}{ }^{h}$ will be seen to be the real representation of symplectic gsroup $S p(n)$. We call the group the symplectic group of $V$ and its element a symplectic transformation of $V$ for brevity. We define the tensors $\kappa_{i}{ }^{h}, \phi_{i h}, \psi_{i_{h}}$ and $\kappa_{i h}$ by

It is easily seen that

$$
\kappa_{i}^{h}=\phi_{k}{ }^{h} \psi_{i}{ }^{k}, \quad \phi_{i h}=\phi_{i}^{a} g_{a_{h}}, \quad \psi_{i_{h}}=\psi_{i}{ }^{a} g_{a_{h}}, \quad \kappa_{i h}=\kappa_{i}^{a} g_{a h} .
$$

$$
\begin{aligned}
& \kappa_{k}{ }^{h} \kappa_{i}{ }^{k}=-\delta_{i}{ }^{h}, \quad \psi_{k}{ }^{l} \kappa_{i}{ }^{k}=-\kappa_{k}{ }^{h} \psi_{i}{ }^{k}=\phi_{i}{ }^{h}, \\
& \kappa_{i}{ }^{h} \phi_{i}{ }^{k}=-\phi_{k}{ }^{h} \kappa_{i}{ }^{k}=\psi_{i}{ }^{h}, \quad \kappa_{i h}+\kappa_{h i}=0 .
\end{aligned}
$$

We denote the linear transformations corresponding to $\phi_{i}{ }^{h}, \psi_{i}{ }^{h}$ and $\kappa_{i}{ }^{h}$ by $I, J$ and $K$ respectively. Since we have

$$
\phi_{i}{ }^{b} \phi_{h l}{ }^{a} g_{b a}=g_{i h h}, \quad \boldsymbol{\psi}_{i}{ }^{b} \boldsymbol{\psi}_{h}{ }^{a} g_{b a}=g_{i h} \quad \text { and } \quad \kappa_{i}{ }^{b} \kappa_{h}{ }^{a} g_{b a}=g_{i_{h}},
$$

$I, J$ and $K$ are orthogonal transformations. Let us suppose that a set of vectors $\left\{x_{1}, I x_{1}, J x_{1}, K x_{1}, x_{2}, I x_{2}, J x_{2}, K x_{2}, \ldots, x_{m}, I x_{m}, J x_{m}, K x_{m}, y\right\}$ is orthonormal. Then so is the set $\left\{x_{1}, I x_{1}, J x_{1}, K x_{1}, \ldots, x_{m}, I x_{m}, J x_{m}, K x_{m}, y, I y, J y\right.$,

1) Numbers in brackets refer to the bibliography at the end of the paper. 
$K y$ \}. In fact, $I y, \cdot J y$ and $K y$ are unit vectors and we have

$$
\begin{aligned}
& g_{i a} y^{i} \phi_{h}{ }^{n} y^{h}=\phi_{h i} y^{i} y^{h}=0 \text {, } \\
& g_{i a} y^{i} \psi_{h}{ }^{a} y^{h}=\psi_{h i} y^{i} y^{h}=0 \\
& g_{i a} y^{i} \kappa_{h}{ }^{a} y^{h}=\kappa_{h} i y^{i} y^{h}=0 \text {, } \\
& g_{s r} \phi_{h l}^{s} y^{h} \psi_{i}{ }^{r} y^{i}=-g_{s r} \phi_{h}{ }^{s} \phi_{a^{r}} \kappa_{i}{ }^{i} y^{h} y^{i}=-g_{h a} \kappa_{i}{ }^{a} y^{h} y^{b}=-\kappa_{i h} y^{i} y^{h}=0, \\
& g_{s r} \phi_{h}{ }^{s} y^{h} \kappa_{i}{ }^{r} y^{i}=g_{s r} \phi_{h}{ }^{s} \phi_{a}{ }^{r} \psi_{i}{ }^{a} y^{h} y^{i}=g_{h a} \psi_{i} a_{y^{h} y^{i}}=\psi_{i h} y^{i} y^{h}=0 \text {, } \\
& g_{s r} \psi_{h}^{s} y^{h} \kappa_{i}^{r} y^{i}=-g_{s r} \psi_{h}{ }^{s} \psi_{a}^{r} \phi_{i}{ }^{a} y^{h} y^{b}=-g_{h} a \phi_{i} y_{y^{h}} y^{i}=-\phi_{i h} y^{k} y^{h}=0 \text {, } \\
& g_{a b} x_{\alpha}{ }^{\prime} \phi_{h}{ }^{a} y_{*}^{h}=g_{i j} \phi_{a}{ }^{i} \phi_{b}{ }^{j} x_{\alpha}^{a} \phi_{h}{ }^{b} y^{h}=-g_{i h} \phi_{a^{i}} x_{\alpha}^{a} y^{h}=0 \text {, } \\
& g_{b a} \phi_{i}{ }^{b} x_{\alpha}^{i} \phi_{l}{ }^{a} y^{h}=g_{i h} x_{\alpha}^{i} y^{h}=0 \text {, } \\
& g_{s r} \psi_{a}{ }^{s} x_{a}^{a} \phi_{h}{ }^{r} y^{h}=g_{i j} \phi_{r}{ }^{j} \phi_{s}{ }^{i} \psi_{a}{ }^{s} x_{a}^{a} \phi_{h l}{ }^{r} y^{h}=-g_{i_{h}} \kappa a^{i} x_{\alpha}^{a} y^{h}=0 \text {, } \\
& g_{s r} \kappa_{a}{ }^{s} x_{\alpha}^{a} \phi_{h}{ }^{r} y^{h}=g_{i j} \phi_{r^{j}} \phi_{s}{ }^{i} \kappa_{a}^{s} x_{a}^{a} \phi_{h}{ }^{r} y^{h}=g_{i h} \psi_{a}{ }^{i} x_{\alpha}^{a} y^{h}=0,
\end{aligned}
$$

and similarly

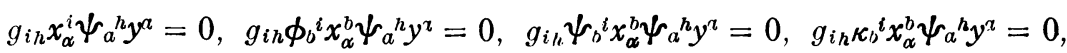

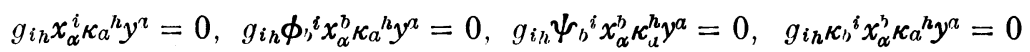

$$
\begin{aligned}
& (\alpha=1,2, \ldots, m) \text {. }
\end{aligned}
$$

It follows that for any unit veztor $e$ there exists an orthonormal base $\left\{e_{1}\right.$, $\left.\ldots, e_{n}, J e_{1}, \ldots, I e_{n}, I e_{1}, \ldots, I e_{n}, K e_{1}, \ldots, K e_{n}\right\}$ of $V$ such that $e_{1}=e$.

Let $\left\{e_{1}, \ldots, e_{n}, J e_{1}, \ldots, J e_{n}, I e_{1}, \ldots, I e_{n}, K e_{1}, \ldots, K e_{n}\right\}$ be an orthonormal base of $V$ and $\sigma$ be a symplectic transformation of $V$. If we have $\sigma e_{\alpha}=f_{\alpha}{ }^{2)}$, then $\sigma J e_{\alpha}=J \sigma e_{\alpha}=J f_{\alpha}, \quad \sigma I e_{\alpha}=I \sigma e_{\alpha}=I f_{\alpha}, \sigma K e_{\alpha}=K \sigma e_{\alpha}=$ $K f_{\alpha}$. Therefore $\sigma$ transforms any orthonormal base $\left\{e_{1}, \ldots, e_{n}, J e_{1}, \ldots\right.$, $\left.J e_{n}, I e_{1}, \ldots, I e_{n}, K e_{1}, \ldots, K o_{n}\right\}$ into an orthonor.nal base $\left\{f_{1}, \ldots, f_{n}, J f_{1}\right.$, $\left.\ldots, I f_{n}, I f_{1}, \ldots, I f_{n}, K f_{1}, \ldots, K f_{n}\right\}$. Conversely, the linear transformation of $V$ which transforms an orthonormal base $\left\{e_{1} \ldots, e_{n}, J e_{1}, \ldots, J e_{n}, I e_{1}, \ldots\right.$, $\left.I e_{n}, K e_{1}, \ldots, K e_{n}\right\}$ into an orthonormal base $\left\{f_{1}, \ldots, f_{n}, J f_{1}, \ldots J f_{n}\right.$, $\left.I f_{1}, \ldots, I f_{n}, K f_{1}, \ldots, K f_{n}\right\}$ is a symplectic transformation of $V$. We see immediately that the components of tensors $g_{i h}, \phi_{i}{ }^{h}, \psi_{i}{ }^{h}, \kappa_{i}{ }^{h}$ with respect to an orthonormal base $\left\{e_{1}, \ldots, e_{n}, J e_{1}, \ldots, J e_{n}, I e_{1}, \ldots, I e_{n}, K e_{1}, \ldots, K e_{n}\right\}$ are the elenents in $h$-th row and $i$-th column of the following four matrices respectively

$$
\left(E_{4 n}\right), \quad\left(\begin{array}{ll}
0-E_{2 n} \\
E_{\underline{y} n} & 0
\end{array}\right), \quad\left(\begin{array}{cccc}
0 & -E_{n} & 0 & 0 \\
E_{n} & 0 & 0 & 0 \\
0 & 0 & 0 & E_{n} \\
0 & 0 & -E_{n} & 0
\end{array}\right), \quad\left(\begin{array}{llll}
0 & 0 & 0 & -E_{n} \\
0 & 0 & E_{n} & 0 \\
0 & -E_{n} & 0 & 0 \\
E_{n} & 0 & 0 & 0
\end{array}\right),
$$

where $E_{q n}, E_{2 n}$ and $E_{n}$ are unit matrices of degrees $4 n, 2 n$, and $n$ respectively. This shows that the symplectic group of $V$ is the real representation of symplectic group $S p(n)$.

2) Throughout the paper Greek indices $\alpha, \beta, \ldots \ldots$ run over the range $1,2, \ldots \ldots, n$ and Latin indices $h, i, j, \ldots \ldots$ over the range $1,2, \ldots \ldots 4 n$. 
The subspace spanned by a set of vectors $\{x, J x, I x, K x\}$ is called the $\boldsymbol{Q}$-section determined by the vector $x$ and is denoted by $\boldsymbol{Q}(x)$.

2. We now state the following

THEOREM 1. Let the tensors $v_{i}, h_{j i}, T_{j i}{ }^{h}$ and $R_{i j i}{ }^{h}=-R_{j k i}{ }^{h}$ be invariant under the symplectic group of $V(\operatorname{dim} V=4 n)$. Then

(1. a) $v_{i}=0$,

(1. b) $\quad h_{j i}=k_{0} g_{j i}+k_{1} \phi_{j i}+k_{2} \psi_{j i}+k_{3} \kappa_{j i}$,

(1. c) $\quad T_{i i}^{h}=0$ for any $n$,

$$
\begin{aligned}
& R_{k j i}{ }^{h}=\left(c_{1} \delta_{a}{ }^{h}+c_{2} \phi_{a^{h}}+c_{3} \psi_{a^{h}}+c_{4} \kappa_{a}{ }^{h}\right)\left(\delta_{k}{ }^{n} g_{j i}-\delta_{j}{ }^{a} g_{k i}\right) \\
& +\left(c_{5} \delta_{a^{h}}+c_{5} \phi_{a^{h}}+c_{7} \psi_{a^{h}}+c_{8} \kappa a^{h}\right)\left(\delta_{i i}^{a} \phi_{j i}-\delta_{j}{ }^{a} \phi_{k i}\right) \\
& +\left(c_{9} \delta_{a^{h}}+c_{10} \phi_{a^{h}}+c_{11} \boldsymbol{\psi}_{a^{h}}+c_{12} \kappa^{h}\right)\left(\delta_{i i}{ }^{a} \boldsymbol{\psi}_{j i}-\delta_{j}{ }^{a} \boldsymbol{\psi}_{k i}\right) \\
& +\left(c_{13} \delta_{a}^{h}+c_{14} \phi_{a^{h}}+c_{15} \psi_{a^{h}}+c_{15} \kappa a^{h}\right)\left(\delta_{k}{ }^{a} \kappa_{j i}-\delta_{j}{ }^{a} \kappa_{k i}\right) \\
& +\left(c_{17} \delta_{i}^{h}+c_{18} \phi_{i}{ }^{h}+c_{19} \psi_{i}{ }^{h}+c_{20} \kappa_{i}{ }^{h}\right) \phi_{i j} \\
& +\left(c_{21} \delta_{i}{ }^{h}+c_{22} \phi_{i}{ }^{h}+c_{23} \psi_{i}{ }^{h}+c_{24} \kappa_{i}{ }^{h}\right) \psi_{k j} \\
& +\left(c_{25} \delta_{i}{ }^{h}+c_{25} \phi_{i}{ }^{h}+c_{27} \psi_{i}{ }^{h}+c_{28} \kappa_{i}{ }^{h}\right) \kappa_{k j} \quad \text { for } n \neq 1 \text {, }
\end{aligned}
$$

where k's and c's are constants.

$$
\text { If moreover } R_{[k i i]}^{h}=0 \text {, then we have in (1.d) }
$$

(1. e) $\quad 2 c_{5}+c_{17}=2 c_{5}+c_{18}=2 c_{7}+c_{19}=2 c_{8}+c_{20}=2 c_{9}+c_{21}=2 c_{10}+c_{22}$ $=2 c_{11}+c_{23}=2 c_{12}+c_{24}=2 c_{13}+c_{25}=2 c_{14}+c_{26}=2 c_{15}+c_{27}=2 c_{16}+c_{28}=0$.

If $R_{k j h h}{ }^{a} g_{i a}+R_{k j i}{ }^{a} g_{a h}=0$, then we have in (1.d)

(1. f) $\quad c_{2}-c_{5}=c_{3}-c_{9}=c_{4}-c_{13}=c_{7}-c_{10}=c_{5}-c_{14}$

$$
=c_{12}-c_{15}=c_{17}=c_{21}=c_{25}=0 .
$$

If $R_{k j l}{ }^{h} \phi_{i}{ }^{a}-R_{k j i}{ }^{a} \phi_{a}{ }^{h}=0$, then we have in (1.d)

$$
\begin{aligned}
c_{1}-c_{i 3} & =c_{2}+c_{5}=c_{3}-c_{8}=c_{4}+c_{\tau}=c_{9}-c_{14}=c_{10}+c_{13}=c_{11}-c_{15} \\
& =c_{12}+c_{15}=c_{19}=c_{20}=c_{23}=c_{24}=c_{27}=c_{28}=0 .
\end{aligned}
$$

If $R_{k, j a}{ }^{h} \psi_{i}{ }^{a}-R_{k j i}{ }^{a} \psi_{a}{ }^{h}=0$, then we have in (1.d)

$$
\begin{gathered}
c_{1}-c_{11}=c_{2}+c_{12}=c_{3}+c_{9}=c_{4}-c_{10}=c_{5}+c_{15}=c_{6}-c_{16}=c_{7}-c_{13} \\
=c_{3}+c_{14}=c_{18}=c_{20}=c_{22}=c_{24}=c_{2 i}=c_{28}=0 .
\end{gathered}
$$

PROOF. (1. a) No non-zero vector is invariant under the symplectic group of $V$.

(1. b) If $h_{j i}$ is invariant under a group of linear transformations, then so are the symmetric part $h_{(j i)}$ and the anti-symmetric part $h_{[j i]}$ of $h_{j i}$. We study two cases.

In case $h_{j i}$ is symmetric, we consider a constant $k_{0}$ such that, for one particular unit vector $e_{0}$,

$$
h_{j i} e_{0}^{j} e_{0}^{i}=k_{0} .
$$

The tensor $h_{j i}-k_{0} g_{j i}$ is invariant under the symplectic group of $V$. For any unit vector $e$ there exists a symplectic transformation of $V$ sending $e_{0}$ to $e_{\text {. }}$ 
Therefore

$$
\left(h_{j i}-k_{0} g_{j i}\right) e^{j} e^{i}=\left(h_{j i}-k_{0} g_{j i}\right) e_{0}^{j} e_{0}^{i}=0 .
$$

This implies

$$
h_{j i}=k_{0} g_{j i} .
$$

We next consider the case in which $h_{j i}$ is anti-symmetric. We denote $h_{j i} w^{j} v^{i}$ by $h(w, v)$. Let $\left\{e_{1}, \ldots, e_{n}, J e_{1}, \ldots, J e_{n}, I e_{1}, \ldots, I e_{n}, K e_{1}, \ldots K e_{n}\right\}$ be an orthonormal base of $V$. We denote $J e_{\alpha}$ by $\boldsymbol{e}_{\alpha_{1}}, I e_{\alpha}$ by $\boldsymbol{e}_{\alpha_{2}}$ and $K e_{\alpha}$ by $\boldsymbol{e}_{\alpha_{3}}$. We say that $\alpha, \alpha_{1}(=n+\alpha), \alpha_{2}(=2 n+\alpha)$ and $\alpha_{3}(=3 n+\alpha)$ belong to the same class. The numbers from 1 to $4 n$ separate into $n$ classes. If the class of $i$ is different from that of $j$, then there exists a symplectic transformation sending $e_{i}$ to $e_{j}$ and $e_{j}$ to $e_{i}$. Hence we have $h\left(e_{j}, e_{i}\right)=h\left(e_{i}, e_{j}\right)$. Since $h_{j i}$ is anti-symmetric, $h\left(e_{j}, e_{i}\right)=0$ if the class of $i$ differs from that of $j$.

We shall show that

$$
\begin{aligned}
h\left(e_{\alpha}, e_{\alpha_{1}}\right) & \left.=h\left(e_{\alpha_{3}}, e_{\alpha_{2}}\right)=h^{\prime} e_{\alpha^{\prime}}, e_{\alpha^{\prime}}\right), h\left(e_{\alpha}, e_{\alpha_{2}}\right)=h\left(e_{\alpha_{1}}, e_{\alpha_{3}}\right) \\
& =h\left(e_{\alpha^{\prime}}, e_{\alpha^{\prime}{ }_{2}}\right), \quad h\left(e_{\alpha}, e_{\alpha_{3}}\right)=h\left(e_{\alpha_{2}}, e_{\alpha_{1}}\right)=h\left(e_{\alpha^{\prime}}, e_{\alpha^{\prime}}\right) .
\end{aligned}
$$

The equalities of the type $h\left(e_{j}, e_{i}\right)=h\left(e_{j^{\prime}}, e_{i^{\prime}}\right)$ are obtained by applying those symplectic transformations which send $e_{j}$ to $e_{j^{\prime}}$ and $e_{i}$ to $e_{i^{\prime}}$. We denote by $\sigma_{1}$ the symplectic transformation sending $e_{\alpha}$ to $e_{\alpha_{2}}, e_{\alpha_{1}}$ to $-e_{\alpha_{3}}, e_{\alpha_{2}}$ to $-e_{\alpha}$ and $\boldsymbol{e}_{\alpha_{3}}$ to $\boldsymbol{e}_{\alpha_{1}}$, by $\sigma_{2:}$ the symplectic transformation sending $\boldsymbol{e}_{\alpha}$ to $\boldsymbol{e}_{\alpha_{1}}, \boldsymbol{e}_{\alpha_{1}}$ to $-\boldsymbol{e}_{\alpha}, \boldsymbol{e}_{\alpha_{2}}$ to $\boldsymbol{e}_{\alpha_{3}}$ and $\boldsymbol{e}_{\alpha_{3}}$ to $-\boldsymbol{e}_{\alpha_{2}}$, and by $\sigma_{3}\left(=\sigma_{1} \sigma_{2}\right)$ the symplectic transformation sending $e_{\alpha}$ to $-e_{\alpha_{3}}, e_{\alpha_{1}}$ to $-e_{\alpha_{2}}, e_{\alpha_{2}}$ to $e_{\alpha_{1}}$ and $e_{\alpha_{3}}$ to $e_{\alpha}$. By applying $\sigma_{1}$ we get $h\left(e_{\alpha}, e_{\alpha_{1}}\right)=h\left(e_{\alpha_{3}}, e_{\alpha_{2}}\right)$ and $h\left(e_{\alpha}, e_{\alpha_{3}}\right)=h\left(e_{\alpha_{2}}, e_{\alpha_{1}}\right)$. By applying $\sigma_{2}$ we get $h\left(e_{\alpha}, e_{\alpha_{2}}\right)=h\left(e_{\alpha_{1}}, e_{\alpha_{3}}\right)$.

If we set $\left.t_{j i}=h_{j i}-h^{\prime} e_{\alpha}, e_{\alpha_{2}}\right) \phi_{j i}-h\left(e_{\alpha}, e_{\alpha_{1}}\right) \psi_{j i}-h\left(e_{\alpha}, e_{\alpha_{8}}\right) \kappa_{j i}$, then the tensor $t_{j i}$ is anti-symmetric and invariant under the symplectic group of $V$. We see immediately $t\left(e_{\alpha}, e_{\alpha_{2}}\right)=t\left(e_{\alpha}, e_{\alpha_{1}}\right)=t\left(e_{\alpha}, e_{\alpha_{3}}\right)=0$. Hence $t_{j i}=0$, i. e. there exist three constants $k_{1}, k_{2}, k_{3}$ such that

$$
h_{j i}=k_{1} \phi_{j i}+k_{2} \psi_{j i}+k_{3} \kappa_{j i} \text {. }
$$

Since the tensor $h_{j i}$ is the sum of its symmetric part and antisymmetric part, (1. b) is proved.

(1. c) Let $T_{j i}{ }^{h}$ be invariant under the symplectic group of $V$. Since the linear transformation $-\delta_{i}{ }^{h}$ is a symplectic transformation of $V$, we have

$$
T_{j i}{ }^{h}=-\delta_{j}^{j^{\prime}}\left(-\delta_{i} i^{\prime}\right)\left(-\delta_{h^{\prime}}{ }^{h}\right) T_{j^{\prime} i^{\prime}} h^{\prime}=-T_{j i}{ }^{h},
$$

and hence

$$
T_{j i}^{h}=0 .
$$

3. To prove (1.d) we prove two lemmas, and this section is devoted to the proof of the lemmas. Let $R_{k j i}{ }^{h}=-R_{j k i}{ }^{h}$ be invariant under the symplectic group of $V$. The tensor $R_{k j i}{ }^{h}$ is determined by the bilinear mapping

shortly

$$
\left(f^{k j}, v^{i}\right) \rightarrow R_{k j i}^{h} f^{k j} v^{i}
$$

$$
(f, v) \rightarrow R(f, v)
$$


of pairs of a bivector and a vector, onto vectors.

We take again an orthonormal base $\left\{e_{1}, \ldots, e_{n}, J e_{1}, \ldots, J e_{n}, I e_{1}, \ldots\right.$, $\left.I e_{n}, K e_{1}, \ldots, K e_{n}\right\}$ and consider in the proof of lemmas and (1.d) components of tensors with respect to this base.

LEMMA 1 . The component $R_{k, j i}{ }^{h}$ vanishes unless $h, i, j, k$ belong to the same class or two of them belong to a class and others do to another one.

Proof. We distinguish two cases $\mathrm{A}$ and $\mathrm{B}$.

Case A. $j$ and $k$ are in the same class.

Subcase $1 . \quad i, j$ and $k$ are in the same class. The vector $R\left(e_{k} \wedge e_{j}, e_{i}\right)=$ $\boldsymbol{R}_{k j i}{ }^{h} \boldsymbol{e}_{h}$ is the sum of two orthogonal component vectors in the $\boldsymbol{Q}$-section $\boldsymbol{Q}\left(\boldsymbol{e}_{i}\right)$ determined by $e_{i}$ and in its orthogonal complement. The vector $R\left(e_{k} \wedge e_{j}, e_{i}\right)$ remains fixed for that symplectic transformation which leaves every vector in $Q\left(e_{i}\right)$ fixed and multiplies every vector in the orthogonal complenent of $Q\left(e_{i}\right)$ by -1 . The component vector of $R\left(e_{k} \wedge e_{j}, e_{i}\right)$ in $Q\left(e_{i}\right)$ remains fixed for the symplectic transformation, while the component vector of $R\left(e_{k} \wedge e_{j}, e_{i}\right)$ in the orthogonal complement of $\left.Q^{\prime} \boldsymbol{e}_{i}\right)$ reverses its direction. Thereiore the vector $R\left(e_{k} \wedge e_{j}, e_{i}\right)$ coincides with its component vector in $Q^{\prime}\left(\boldsymbol{e}_{i}\right)$, i. e. $R_{k j i}^{h}=0$ unless the class of $h$ is that of $i$.

Subcase 2. The class of $i$ is different from that of $j$. The vector $R\left(e_{i} \wedge\right.$ $\left.\boldsymbol{e}_{i}, \boldsymbol{e}_{i}\right)$ remains fixed for the symplectic transformation of $V$ leaving fixed every vector in $Q\left(e_{i}\right)$ and $Q\left(e_{j}\right)$ and multiplying every vector orthogonal to both $\boldsymbol{Q}\left(\boldsymbol{e}_{i}\right)$ and $\boldsymbol{Q}\left(\boldsymbol{e}_{j}\right)$ by -1 . It follows from this that $R_{k j i}{ }^{h}=0$ if the class of $h$ is neither that of $i$ nor that of $j$. The symplectic transformation of $V$ multiplying every vector in $Q\left(e_{j}\right)$ by -1 and leaving fixed every vector orthogonal to $Q\left(\boldsymbol{e}_{j}\right)$ leaves the vector $R\left(\boldsymbol{e}_{k} \wedge \boldsymbol{e}_{j}, \boldsymbol{e}_{i}\right)$ fixed. Therefore $R_{k j i} \boldsymbol{l}^{\mathrm{l}}=0$ if the class of $h$ is that of $j$.

Case B. $j$ and $k$ are in different classes.

Subcase 1. The class of $i$ is either that of $j$ or that of $k$. The vector $R\left(\boldsymbol{e}_{k} \wedge \boldsymbol{e}_{j}, \boldsymbol{e}_{i}\right)$ remains fixed for that symplectic transformation which leaves fixed every vector in $Q\left(e_{j}\right)$ and $\boldsymbol{Q} \boldsymbol{e}_{k}$ ) and multiplies every vector orthogonal to both $Q\left(e_{j}\right)$ and $Q\left(e_{k}\right)$ by -1 . It follows irom this that $R_{i v i j}{ }^{h}=0$ if the class of $h$ is neither that of $j$ nor that of $k$. We may assume without loss of generality that the class of $i$ is that of $j$. The symplectic transformation of $V$ multiplying every vector in $Q\left(e_{i}\right)$ by -1 and leaving fixed every vector orthogonal to $Q\left(e_{i}\right)$ leaves the vector $R\left(e_{k} \wedge e_{i}, e_{i}\right)$ fixed. Therefore $R_{i j i}{ }^{h}=0$ if the class of $h$ is that of $i$.

Subcase 2. The class of $i$ is neither that of $j$ nor that of $k$. The vector $R\left(e_{i} \wedge e_{j}, e_{i}\right)$ remains fixed for that symplectic transformation which leaves fixed every vector in $Q^{\prime}\left(e_{i}\right)$ and multiplies every vector orthogonal to $Q\left(e_{i}\right)$ by -1 . Hence $R_{k j i}{ }^{h}=0$ unless the class of $h$ is that of $i$. The vector $R\left(e_{i} \wedge e_{j}\right.$, $\boldsymbol{e}_{i}$ ) remains fixed for that symplectic transformation which multiplies every vector in $Q\left(e_{i}\right)$ and $Q\left(e_{j}\right)$ by -1 and leaves fixed every vector orthogonal to both $Q\left(e_{i}\right)$ and $Q\left(e_{j}\right)$. Therefore $R_{k j i}{ }^{h}=0$ if the class of $h$ is that of $i$. This completes the proof of lemma 1 . 
LEMMA 2. Let the dimension of $V$ be greater than 4. Then the tensor $R_{k s i}{ }^{h}$ is zero if the following 28 components vanish:

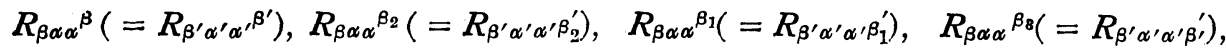

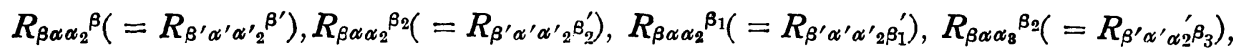

$R_{\beta \alpha \alpha_{1}}{ }^{\beta}\left(=R_{\beta^{\prime} \alpha^{\prime} \alpha^{\prime}{ }_{1}}^{\beta^{\prime}}\right), R_{\beta \alpha \alpha \alpha_{1}}{ }^{\beta_{2}}\left(=R_{\beta^{\prime} \alpha^{\prime} \alpha^{\prime} 1} \beta_{2}^{\prime}\right), R_{\beta \alpha \alpha_{1}}^{\beta_{1}}\left(=R_{\beta^{\prime} \alpha^{\prime} \alpha^{\prime} 1_{1} \beta_{1}^{\prime}}\right), R_{\beta \alpha \alpha_{1}}{ }^{\beta_{8}}\left(=R_{\beta^{\prime} \alpha^{\prime} \alpha^{\prime} \alpha_{3} \beta_{3}^{\prime}}\right)$,

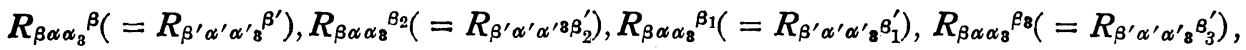

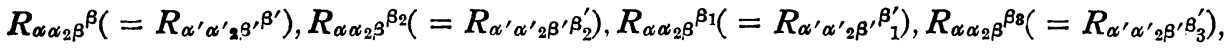

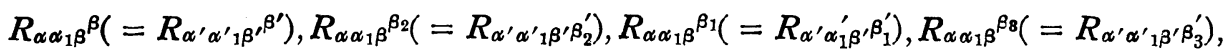

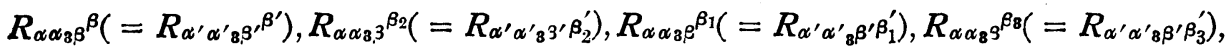

where different letters in the indices of components of the tensor belong to different classes.

PROoF. By the assumption and lemma 1 the vectors $R\left(e_{\beta} \wedge e_{\alpha}, e_{\alpha}\right), R\left(e_{\beta} \wedge\right.$ $\left.\boldsymbol{e}_{\alpha}, \boldsymbol{e}_{\alpha_{2}}\right), R\left(e_{\beta} \wedge e_{\alpha}, e_{\alpha_{1}}\right), R\left(e_{\beta} \wedge e_{\alpha}, e_{\alpha_{3}}\right), R\left(e_{\alpha} \wedge e_{\alpha_{2}}, e_{\beta}\right), R\left(e_{\alpha} \wedge e_{\alpha_{1}}, e_{\beta}\right)$ and $R\left(e_{\alpha} \wedge\right.$ $\left.\boldsymbol{e}_{\alpha_{3}}, \boldsymbol{e}_{\beta}\right)$ are zero. We denote by $\sigma_{1 \alpha}$ the symplectic transformation fixing every vector orthogonal to $Q^{\prime}\left(e_{\alpha}\right)$ and sending $e_{\alpha}$ to $e_{\alpha_{2}}, e_{\alpha_{1}}$ to $-e_{\alpha_{3}}, e_{\alpha_{2}}$ to $-e_{\alpha}$ and $e_{\alpha_{3}}$ to $e_{\alpha_{1}}$. Let us denote by $\sigma_{2 \alpha}$ the symplectic transformation fixing every vector orthogonal to $\boldsymbol{Q}^{\prime}\left(\boldsymbol{e}_{\alpha}\right)$ and sending $\boldsymbol{e}_{\alpha}$ to $\boldsymbol{e}_{\alpha_{1}}, \boldsymbol{e}_{\alpha_{1}}$ to $-\boldsymbol{e}_{\alpha}$, $\boldsymbol{e}_{\alpha_{2}}$ to $\boldsymbol{e}_{\alpha_{3}}$ and $\boldsymbol{e}_{\alpha_{3}}$ to $-e_{\alpha_{2}}$ and by $\sigma_{3 \alpha}\left(=\sigma_{1 \alpha} \sigma_{2 \alpha}\right)$ the symplectic transformation fixing every vector orthogonal to $Q\left(\boldsymbol{e}_{\alpha}\right)$ and sending $\boldsymbol{e}_{\alpha}$ to $-\boldsymbol{e}_{\alpha_{3}}, \boldsymbol{e}_{\alpha_{1}}$ to $-e_{\alpha}, e_{\alpha_{2}}$ to $e_{\alpha_{1}}$ and $e_{\alpha_{3}}$ to $e_{\alpha}$. Applying $\sigma_{1 \alpha}, \sigma_{2 \alpha}, \sigma_{3 \alpha}, \sigma_{1 \beta}, \sigma_{2 \beta}$ or $\sigma_{3,3}$ to the vectors indicated above, we see that the vector $R\left(e_{k} \wedge e_{j}, e_{i}\right)$ is zero if $i, j$ and $k$ are in two classes.

We next consider any vector $R\left(e_{k} \wedge e_{j}, e_{i}\right)$ such that $i, j$ and $k$ belong to the same class, say, that of $\alpha$. Let $\alpha^{\prime}$ be different from $\alpha$. Let us apply to the relation $R\left(e_{k} \wedge e_{j}, e_{i}\right)=R_{k, j i}^{h} e_{h}=R_{k j i \alpha} e_{\alpha}+R_{k j i \alpha_{1}} e_{\alpha_{1}}+R_{k j i \alpha_{2}} e_{\alpha_{2}}+R_{k j i \alpha_{3}} e_{\alpha_{8}}$ such symplectic transformation which sends $e_{\alpha}$ to $\frac{1}{\sqrt{2}}\left(e_{\alpha}+e_{\alpha^{\prime}}\right), e_{\alpha^{\prime}}$ to $\frac{1}{\sqrt{2}}\left(e_{\alpha}-e_{\alpha^{\prime}}\right)$ and hence $e_{\alpha_{1}}$ to $\frac{1}{\sqrt{2}}\left(e_{\alpha_{1}}+e_{\alpha_{1}^{\prime}}\right), e_{\alpha_{2}}$ to $\frac{1}{\sqrt{2}}\left(e_{\alpha_{2}}+e_{\alpha_{2}^{\prime}}^{\prime}\right), e_{\alpha_{3}}$ to $\frac{1}{\sqrt{2}}\left(e_{\alpha_{8}}+e_{\alpha_{3}^{\prime}}^{\prime}\right), e_{\alpha_{1}^{\prime}}^{\prime}$ to $\frac{1}{\sqrt{2}}\left(e_{\alpha_{1}}-e_{\alpha_{1}^{\prime}}\right), e_{\alpha_{2}^{\prime}}$ to $\frac{1}{\sqrt{2}}\left(e_{\alpha_{2}}-e_{\alpha^{\prime} 2}\right)$ and $e_{\alpha^{\prime} 3}$ to $\frac{1}{\sqrt{2}}\left(e_{\alpha_{3}}-e_{\alpha^{\prime} 3}\right)$. Then the vector $R^{\prime}\left(e_{k} \wedge e_{j}, e_{i}\right)$ is transformed into $\frac{1}{2 \sqrt{2}}$ $R\left(\left(e_{k}+e_{k^{\prime}}\right) \wedge\left(e_{j}+e_{j^{\prime}}\right), e_{i}+e_{i}\right)=\frac{1}{2 \sqrt{2}}\left(R\left(e_{k} \wedge e_{j}, e_{i}\right)+R\left(e_{k^{\prime}} \wedge e_{j^{\prime}}, e_{i^{\prime}}\right)\right), \quad$ while the vector $R_{k j i}^{h} e_{h}$ into $\frac{1}{\sqrt{2}} R_{k j i}{ }^{h}\left(e_{h}+e_{h^{\prime}}\right)$. Therefore we have $\frac{1}{2} R\left(e_{k} \wedge e_{j}, e_{i}\right)$ $=R_{k j i}{ }^{h} e_{h}$. Thus the vector $R\left(e_{i} \wedge e_{j}, e_{i}\right)$ is zero if $i, j$ and $k$ belong to the same class. This completes the proof of lemma 2.

4. On the basis of lemmas 1 and 2 we now proceed to the proof of (1. d). We define 28 tensors ${\underset{a}{k j i}}^{h}=-R_{a k i}{ }^{h}(a=1,2, \ldots, 28)$ invariant under 
the symplectic group of $V$ to be those in (1.d) with coefficients $c_{a}$. The components of tensors $g_{i h}, \phi_{i}{ }^{h}, \psi_{i}{ }^{h}, \kappa_{i}{ }^{h}$ with respect to an orthonormal base $\left\{e_{1}, \ldots, e_{n}, J e_{1}, \ldots, J e_{n}, I e_{1}, \ldots, I e_{n}, K e_{1}, \ldots, K e_{n}\right\}$ can be found in section 1. We see that $a$-th component in lemma 2 of the tensor $R_{a j i}{ }^{1}$ is equal to 1 and its other components in lemma 2 are zero. If we define the tensor $T_{k j i}{ }^{h}=-T_{j k i}{ }^{h}$ as follows

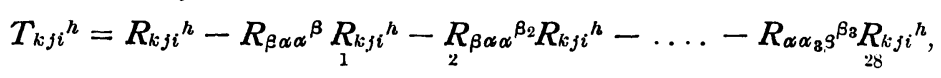

then the tensor is invariant under the symplectic group of $V$ and its 28 components in lemma 2 are zero. Lemma 2 shows that $T_{k i j i}{ }^{h}=0$, i. e. there exist 28 constants $c_{a}(a=1, \ldots, 28)$ such that

Hence (1. d) is proved.

$$
R_{k j i}{ }^{h}=c_{1} R_{k j i}{ }^{h}+c_{2} R_{2 k j i}{ }^{h}+\ldots+c_{25} R_{28}{ }^{h j i} .
$$

(1. e) By alternation of (1.d) we obtain

$$
\begin{aligned}
& 3 \boldsymbol{R}_{[k j i]}{ }^{h}=\left(2 c_{5}+c_{17}\right)\left(\delta_{k}{ }^{n} \phi_{j i}+\delta_{i}{ }^{h} \phi_{k j}+\delta_{j}{ }^{h} \phi_{i k}\right) \\
& +\left(2 c_{6}+c_{18}\right)\left(\phi_{k i}{ }^{\prime \prime} \phi_{j i}+\phi_{i}{ }^{l} \phi_{k j}+\phi_{j}{ }^{h} \phi_{i k}\right) \\
& +\left(2 c_{7}+c_{19}\right)\left(\psi_{k i}{ }^{\prime \prime} \phi_{j i}+\psi_{i}{ }^{h} \phi_{k j}+\psi_{j}{ }^{h} \phi_{i k}\right) \\
& +\left(2 c_{8}+c_{20}\right)\left(\kappa_{k}{ }^{l} \phi_{j i}+\kappa_{i}{ }^{h} \phi_{k j}+\kappa_{j}{ }^{h} \phi_{i k}\right) \\
& +\left(2 c_{9}+c_{21}\right)\left(\delta_{k}{ }^{h} \psi_{j i}+\delta_{i}{ }^{h} \psi_{k j}+\delta_{j}{ }^{h} \psi_{i k}\right) \\
& +\left(2 c_{10}+c_{22,}\right)\left(\phi_{k i}{ }^{h} \boldsymbol{\psi}_{j i}+\phi_{i}{ }^{h} \boldsymbol{\psi}_{k j}+\phi_{j}{ }^{h} \boldsymbol{\psi}_{i k}\right) \\
& +\left(2 c_{11}+c_{23}\right)\left(\psi_{k i}{ }^{\prime \prime} \psi_{j i}+\psi_{i}{ }^{\prime \prime} \psi_{k j}+\psi_{j}{ }^{\prime \prime} \psi_{i k}\right) \\
& +\left(2 c_{12}+c_{24}\right)\left(\kappa_{k}^{h} \psi_{j i}+\kappa_{i}^{h} \psi_{k j}+\kappa_{j}^{h} \psi_{i k}\right) \\
& +\left(2 c_{13}+c_{25}\right)\left(\delta_{k}{ }^{h} \kappa_{j i}+\delta_{i}{ }^{h} \kappa_{k j}+\delta_{j}{ }^{h} \kappa_{i k}\right) \\
& +\left(2 c_{14}+c_{2 j}\right)\left(\phi_{k i}{ }^{h} \kappa_{j i}+\phi_{i}{ }^{h} \kappa_{k j}+\phi_{j}{ }^{h} \kappa_{i k}\right) \\
& +\left(2 c_{15}+c_{27}\right)\left(\psi_{k i}{ }^{h} \kappa_{j i}+\psi_{i}{ }^{h} \kappa_{k j}+\psi_{j}{ }^{h} \kappa_{i k}\right) \\
& +\left(2 c_{16}+c_{28}\right)\left(\kappa_{k}{ }^{h} \kappa_{j i}+\kappa_{i}{ }^{h} \kappa_{k j}+\kappa_{j}{ }^{h} \kappa_{i k}\right) \text {. }
\end{aligned}
$$

Considering the components with respect to an orthonormal base $\left\{\boldsymbol{e}_{1}, \ldots\right.$, $\left.e_{n}, J e_{1}, \ldots, J e_{n}, I e_{1}, \ldots, I e_{n}, K e_{1}, \ldots, K e_{n}\right\}$, we see that the tensors in the parentheses are linearly independent. Therefore the condition $R_{\left[\left.k j i\right|^{h}\right.}{ }^{n}=0$ implies $2 c_{5}+c_{17}=2 c_{6}+c_{18}=2 c_{7}+c_{19}=2 c_{8}+c_{20}=2 c_{9}+c_{21}=2 c_{10}+c_{22}=2 c_{11}$ $+c_{23}=2 c_{12}+c_{24}=2 c_{13}+c_{25}=2 c_{14}+c_{26}=2 c_{15}+c_{27}=2 c_{16}+c_{28}=0$.

(1.f) From the relation (1.d) we get

$$
\begin{aligned}
R_{k j h}{ }^{a} g_{i a}+R_{k j i}{ }^{a} g_{a h} & =\left(c_{2}-c_{5}\right)\left(\phi_{k i} g_{j h}-\phi_{j i} g_{k h}+\phi_{k h} g_{j i}-\phi_{j h} g_{k i}\right) \\
& +\left(c_{3}-c_{9}\right)\left(\psi_{k i} g_{j h}-\psi_{j i} g_{k h}+\psi_{k h} g_{j i}-\psi_{j h} g_{k i}\right) \\
& +\left(c_{1}-c_{13}\right)\left(\kappa_{h i} g_{j h}-\kappa_{j i} g_{k h}+\kappa_{k h} g_{j i}-\kappa_{j h} g_{k i}\right) \\
& +\left(c_{7}-c_{10}\right)\left(\psi_{k i} \phi_{j h}-\psi_{j i} \phi_{k h}+\psi_{k h} \phi_{j i}-\psi_{j h} \phi_{k i}\right) \\
& +\left(c_{8}-c_{14}\right)\left(\kappa_{k i} \phi_{j h}-\kappa_{j i} \phi_{k h}+\kappa_{k h} \phi_{j i}-\kappa_{j h} \phi_{k i}\right) \\
& +\left(c_{12}-c_{15}\right)\left(\kappa_{k i} \psi_{j h}-\kappa_{j i} \psi_{k h}+\kappa_{k h} \psi_{j i}-\kappa_{j h} \psi_{k i}\right) \\
& +2 c_{17} g_{i h} \phi_{k j}+2 c_{21} g_{i h} \psi_{k j}+2 c_{25} g_{i h h} \kappa_{k j} .
\end{aligned}
$$


The condition $R_{k, j h}{ }^{a} g_{i a}+R_{k j i j}{ }^{a} g_{a h}=0$ implies $c_{2}-c_{5}=c_{3}-c_{9}=c_{4}-c_{13}$ $=c_{7}-c_{10}=c_{8}-c_{14}=c_{12}-c_{15}=c_{19}=c_{i 1}=c_{25}=0$.

(1.g) From the relation (1.d) we obtain

$$
\begin{aligned}
& R_{k j a}{ }^{h} \phi_{i}{ }^{a}-R_{k j i}{ }^{a} \phi_{a}{ }^{h}=\left(c_{1}-c_{6}\right)\left(\delta_{k}{ }^{h} \phi_{i j}-\delta_{j}{ }^{h} \phi_{i k}-\phi_{k}{ }^{h} g_{j i}+\phi_{j}{ }^{h} g_{k i}\right) \\
& +\left(c_{i 2}+c_{5}\right)\left(\phi_{k}{ }^{\prime \prime} \phi_{i j}-\phi_{j}{ }^{h} \phi_{i k}+\delta_{k i}{ }^{h} g_{j i}-\delta_{j}{ }^{h} g_{k i}\right) \\
& +\left(c_{3}-c_{8}\right)\left(\psi_{\kappa i}{ }^{\prime \prime} \phi_{i j}-\psi_{j}{ }^{h} \phi_{i k}-\kappa_{i:}{ }^{h} g_{j i}+\kappa_{j}{ }^{h} g_{k i}\right) \\
& +\left(c_{4}+c_{7}\right)\left(\kappa_{k}{ }^{\prime \prime} \phi_{i j}-\kappa_{j}{ }^{\prime \prime} \phi_{i k}+\psi_{i}{ }^{h} g_{j i}-\psi_{j}{ }^{h} g_{k i}\right) \\
& +\left(c_{9}-c_{14}\right)\left(\delta_{i}{ }^{h} \kappa_{i j}-\delta_{j}{ }^{h} \kappa_{i k}-\phi_{k}{ }^{\prime} \psi_{j i}+\phi_{j}{ }^{h} \psi_{k i}\right) \\
& +\left(c_{10}+c_{13}\right)\left(\phi_{k}{ }^{h} \kappa_{i j}-\phi_{j}{ }^{h} \kappa_{i k}+\delta_{k}{ }^{h} \psi_{j i}-\delta_{j}{ }^{h} \psi_{k i}\right) \\
& +\left(c_{11}-c_{16}\right)\left(\boldsymbol{\psi}_{i i}{ }^{h} \kappa_{i j}-\boldsymbol{\psi}_{j}{ }^{h} \kappa_{i k}-\kappa_{i}{ }^{h} \boldsymbol{\psi}_{j i}+\kappa_{j}{ }^{h} \boldsymbol{\psi}_{k i}\right) \\
& +\left(c_{1,2}+c_{15}\right)\left(\kappa_{i}^{h} \kappa_{i, j}-\kappa_{j}{ }^{h} \kappa_{i k}+\psi_{k}{ }^{h} \psi_{j i}-\psi_{j}{ }^{h} \psi_{k i}\right) \\
& -2 c_{19} \kappa_{i}{ }^{\prime \prime} \phi_{i j j}+2 c_{\iota 0} \psi_{i}{ }^{\prime \prime} \phi_{i j j} \\
& -2 c_{23} \kappa_{i}{ }^{l} \psi_{k j j}+2 c_{24} \psi_{i}{ }^{l} \psi_{i, j} \\
& -2 c_{27} \kappa_{i}{ }^{i h} \kappa_{k j}+2 c_{28} \psi_{i}{ }^{h} \kappa_{k j} \text {. }
\end{aligned}
$$

Hence the condition $R_{i j j a}{ }^{h} \phi_{i}{ }^{a}-R_{k j i}{ }^{a} \phi_{a^{l i}}=0$ implies $c_{1}-c_{6}=c_{2}+c_{5}=c_{3}-c_{8}$ $=c_{4}+c_{7}=c_{9}-c_{14}=c_{10}+c_{13}=c_{11}-c_{16}=c_{12}+c_{15}=c_{19}=c_{20}=c_{23}=c_{24}=c_{27}$ $=c_{28}=0$.

(1. h) From the relation (1.d) we obtain

$$
\begin{aligned}
& R_{k j, j a}{ }^{h} \boldsymbol{\psi}_{i}{ }^{a}-R_{k j i}{ }^{a} \boldsymbol{\psi}_{a}{ }^{h}=\left(c_{1}-c_{11}\right)\left(\delta_{k}{ }^{h} \boldsymbol{\psi}_{i j}-\delta_{j}{ }^{h} \boldsymbol{\psi}_{i k}-\boldsymbol{\psi}_{k}{ }^{h} g_{j i}+\boldsymbol{\psi}_{j}{ }^{h} g_{k i}\right) \\
& +\left(c_{2}+c_{1:}\right)\left(\phi_{i:}{ }^{h} \psi_{i j}-\phi_{j}{ }^{h} \psi_{i k}+\kappa_{k i}{ }^{h} g_{j i}-\kappa_{j}{ }^{h} g_{k i}\right) \\
& +\left(c_{3}+c_{9}\right)\left(\psi_{k}{ }^{h} \psi_{i j}-\psi_{j}{ }^{h} \psi_{i k}+\delta_{k}{ }^{h} g_{j i}-\delta_{j}{ }^{h} g_{k i}\right) \\
& +\left(c_{1}-c_{10}\right)\left(\kappa_{k}{ }^{\prime \prime} \psi_{i j}-\kappa_{j}{ }^{l} \psi_{i k}-\phi_{k}{ }^{h} g_{j i}+\phi_{j}{ }^{h} g_{k i}\right) \\
& +\left(c_{5}+c_{15}\right)\left(-\delta_{k}{ }^{h} \kappa_{i j}+\delta_{j}{ }^{h} \kappa_{i k}-\psi_{k}{ }^{\prime} \phi_{j i}+\psi_{j}{ }^{h} \phi_{i i}\right) \\
& +\left(c_{5}-c_{16}\right)\left(-\phi_{i}{ }^{\prime \prime} \kappa_{i j}+\phi_{j}{ }^{h} \kappa_{i k}+\kappa_{i k}{ }^{h} \phi_{j i}-\kappa_{j}{ }^{h} \phi_{i i i}\right) \\
& +\left(c_{7}-c_{13}\right)\left(-\psi_{k}{ }^{h} \kappa_{i j}+\psi_{j}{ }^{h} \kappa_{i k}+\delta_{k}{ }^{\prime \prime} \phi_{j i}-\delta_{j}{ }^{h} \phi_{k i}\right) \\
& +\left(c_{8}+c_{14}\right)\left(-\kappa_{k}{ }^{h} \kappa_{i j}+\kappa_{j}{ }^{h} \kappa_{i k}-\phi_{k}{ }^{\prime \prime} \phi_{j i}+\phi_{j}{ }^{h} \phi_{k i}\right) \\
& +2 c_{18} \kappa_{i}{ }^{h} \phi_{k j}-2 c_{20} \phi_{i}{ }^{h} \phi_{k j} \\
& +2 c_{22} \kappa_{i}{ }^{l} \psi_{k_{j}}-2 c_{2_{1}} \phi_{i}{ }^{\prime \prime} \psi_{k j} \\
& +2 c_{20} \kappa_{i}{ }^{h} \kappa_{k j}-2 c_{29} \phi_{i}{ }^{h} \kappa_{k j} \text {. }
\end{aligned}
$$

Hence the condition $R_{k j a}{ }^{h} \psi_{i}{ }^{a}-R_{k j i}{ }^{a} \psi_{a}{ }^{h}=0$ implies $c_{1}-c_{11}=c_{2}+c_{12}=c_{3}+c_{9}$ $=c_{4}-c_{10}=c_{5}+c_{15}=c_{5}-c_{15}=c_{7}-c_{13}=c_{8}+c_{14}=c_{18}=c_{20}=c_{22}=c_{24}=c_{26}$ $=c_{2^{\circ}}=0$.

This completes the proof of all the statement of theorem 1.

If a tensor $R_{k j i}{ }^{h}$ satisfies the conditions $R_{k j a}{ }^{h} \phi_{i}{ }^{a}-R_{k j i}{ }^{a} \phi_{a}{ }^{h}=0$ and $R_{k j a}{ }^{h}$ $\times \psi_{i}^{a}-R_{k j i}{ }^{c} \psi_{a}{ }^{h}=0$, then we have

$$
\begin{aligned}
& R_{k j a}{ }^{h} \kappa_{i}{ }^{a}-R_{k j i}{ }^{a} \kappa_{a}{ }^{h}=R_{k_{j} a}{ }^{h} \phi_{l}{ }^{a} \boldsymbol{\psi}_{i}{ }^{l}-R_{k j i}{ }^{a} \boldsymbol{\phi}_{m}{ }^{h} \boldsymbol{\psi}_{a}{ }^{m} \\
& =\left(\boldsymbol{R}_{\boldsymbol{k} j a}{ }^{h} \boldsymbol{\phi}_{l}{ }^{a}-\boldsymbol{R}_{k j l}{ }^{m} \boldsymbol{\phi}_{m}{ }^{h}, \boldsymbol{\psi}_{i}{ }^{l}+\left(\boldsymbol{R}_{k j a}{ }^{m} \boldsymbol{\psi}_{i}{ }^{a}-\boldsymbol{R}_{k j i}{ }^{a} \boldsymbol{\psi}_{a}{ }^{m}\right) \boldsymbol{\phi}_{m}{ }^{h}\right. \\
& =0 \text {. }
\end{aligned}
$$


If a tensor $R_{k, j i}{ }^{h}=-R_{j k i}{ }^{h}$ invariant under the symplectic group of $V$ satisfies the conditions $R_{\left[k_{j i}\right]^{h}}=0, R_{k j a}{ }^{h} \phi_{i}{ }^{a}-R_{k_{j i}}{ }^{a} \phi_{a}{ }^{h}=0$ and $R_{k j a}{ }^{h} \psi_{i}{ }^{a}-R_{k j i}{ }^{a}$ $\times \psi_{a}{ }^{h}=0$, then theorem 1 shows that $R_{k j i}{ }^{h}=0$.

If a tensor $R_{k j i}{ }^{h}=-R_{j i i}{ }^{h}$ invariant under the symplectic group of $V$ satisfies the conditions $R_{k j h}{ }^{a} g_{i a}+R_{k j i}{ }^{a} g_{a h l}=0, R_{k j a}{ }^{h} \phi_{i}{ }^{a}-R_{k j i}{ }^{a} \phi_{a}{ }^{h}=0$ and $R_{k j a}{ }^{h}$ $\times \psi_{i}{ }^{a}-R_{k j i} \psi^{a} a^{b}=0$, then the tensor has the form

$$
\begin{aligned}
R_{k j i}{ }^{h}=c\left(\delta_{k}{ }^{h} g_{j i}-\delta_{j}{ }^{h} g_{k i}\right. & +\phi_{k}{ }^{h} \phi_{j i}-\phi_{j}{ }^{h} \phi_{k i} \\
& \left.+\psi_{k}{ }^{h} \psi_{j i}-\psi_{j}{ }^{h} \psi_{k i}+\kappa_{k}{ }^{h} \kappa_{j i}-\kappa_{j}{ }^{h} \kappa_{k i}\right) .
\end{aligned}
$$

5. TheOREM 2. Let $M$ be a 4 -dimensional manifold for $n \neq 1$ admitting a quaternion structure $\left(\phi_{i}{ }^{h}, \psi_{i}{ }^{h}\right)$ and an almost Hermitian metric $g_{i_{i}}$ with respect to both $\phi_{i}{ }^{h}$ and $\psi_{i}{ }^{h}$ [3]. Let $\Gamma_{j i}^{h}$ be a $(\phi, \psi)$-connection in $M$ [3]. If the manifold $M$ admits a group of affine transformations and the isotropy group in the tangent space at any point contains the symplectic group, then the connection is locally flat.

PROOF. The value of the torsion tensor field at any point is invariant under the linear isotropy group at the point which contains the symplectic group. It follows from theorem 1 (1.c) that the torsion tensor is zero. Since $\Gamma_{j i}^{h}$ is a $(\phi, \psi)$-connection without torsion, the curvature tensor field $R_{k j i}{ }^{h}$ satisfies the relations $R_{[k j i]^{h}}{ }^{h}=0, R_{k j a}{ }^{h} \phi_{i}{ }^{a}-R_{k j i}{ }^{a} \phi_{a}{ }^{h}=0$ and $R_{k j a}{ }^{h} \psi_{i}{ }^{a}-R_{k j i}{ }^{a} \psi_{a}{ }^{h}$ $=0$. The value of the curvature tensor field at any point is invariant under the symplectic group. Therefore the curvature tensor is zero.

THEOREM 3. Let $M$ be a connected $4 n$-dimensional manifold for $n \neq 1$ admitting a quaternion structure $\left(\phi_{i}{ }^{a}, \psi_{i}{ }^{a}\right)$ and an almost Hermitian metric $g_{i h}$ with respect to both $\phi_{i}{ }^{a}$ and $\psi_{i}{ }^{a}$. Let $G$ be $a\left(2 n^{2}+5 n\right)$-dimensional effective group of automorphisms of $M$. Then the group $G$ is transitive and the LeviCivita's connection is the only linear connection invariant under the group $G$. The connection is $a(\phi, \psi)$-connection and locally flat.

PROof. The linear group of isotropy at any point coincides with the symplectic group of the tangent space at the point. By the same arguments as in the proof of Theorem I in [4] we can conclude that the group $G$ is transitive. Let $\Gamma_{j i}^{h}$ and ' $\Gamma_{j i}^{h}$ be two linear connections in $M$ invariant under the group $G$. The tensor $T_{j i}^{h}=\Gamma_{j i}^{h}-{ }^{\prime} \Gamma_{j i}^{h}$ is invariant under the group $G$. From (1.c) we have $T_{3 i}{ }^{n}=0$. Consequently the Levi-Civita's connection is the only connection invariant under the group. The covariant derivatives of $\phi_{i}{ }^{h}$ and $\psi_{i}{ }^{h}$ are invariant under the group. It follows from (1.c) and theorem 2 that the connection is a $(\phi, \psi)$-connection and locally flat.

THEOREM 4. Let $M$ be a 4n-dimensional manifold for $n \neq 1$ admitting $a$ quaternion structure $\left(\phi_{i}{ }^{h}, \psi_{i}{ }^{h}\right)$ and an almost Hermitian metric $g_{i h}$ with respect to both $\phi_{i}{ }^{h}$ and $\psi_{i}{ }^{h}$. Let $\Gamma_{j i}^{h}$ be a metric $(\phi, \psi)$-connection [3]. We assume that the homogeneous holonomy group of $M$ at a point of $M$ is the symplectic group. If the curvature tensor field has null covariant derivatives, then it has the form

$$
R_{k j i}{ }^{h}=c\left(\delta_{k}{ }^{h} g_{j i}-\delta_{j}{ }^{h} g_{k i}+\phi_{k}{ }^{h} \phi_{j i}-\phi_{j}{ }^{h} \phi_{k i}+\psi_{k}{ }^{h} \psi_{j i}-\psi_{j}{ }^{h} \psi_{k j}+\kappa_{k}{ }^{h} \kappa_{j i}-\kappa_{i}{ }^{h} \kappa_{k i}\right)
$$


with an absolute constant $c$.

PROoF. Since the curvature tensor field has null covariant derivatives, the value of the tensor field at any point is invariant under the homogeneous holonomy group at the point which is the symplectic group. Since $\Gamma_{j i}^{h}$ is a metric $(\phi, \psi)$-connection, the curvature tensor field satisfies the relations $R_{k j h}{ }^{a} g_{i a}+R_{k j i}{ }^{a} g_{a h}=0, R_{k j a}{ }^{h} \phi_{i}{ }^{a}-R_{k j i}{ }^{a} \phi_{a}{ }^{h}=0$ and $R_{k j a}{ }^{h} \psi_{i}{ }^{a}-R_{k j i}{ }^{a} \psi_{a}{ }^{h}=0$. Therefore the tensor field has the form

$$
R_{k j i}{ }^{h}=c\left(\delta_{k}{ }^{h} g_{j i}-\delta_{j}{ }^{h} g_{k i}+\phi_{k}{ }^{h} \phi_{j i}-\phi_{j}{ }^{h} \phi_{k i}+\psi_{k}{ }^{h} \psi_{j i}-\psi_{j}{ }^{h} \psi_{k i}+\kappa_{k}{ }^{h} \kappa_{j i}-\kappa_{j}{ }^{h} \kappa_{k i}\right)
$$

with a scalar $c$. The tensor field in the parentheses is parallel. Hence the scalar $c$ is an absolute constant.

By analogous arguments we have the following [1]

THEOREM 5. Let $M$ be a 2n-dimensional almost Hermitian manifold for $n \neq 1$ and $\Gamma_{j i}^{h}$ be a metric $\phi$-connection. We assume that the homogeneous holonomy group of $M$ at a point of $M$ is the real representation of unitary group. If the curvature tensor field has null covariant derivative, then it has the form

$$
R_{k j i}{ }^{h}=c\left(\delta_{k}{ }^{h} g_{j i}-\delta_{j}{ }^{h} g_{k i}+\phi_{k}{ }^{\prime \prime} \phi_{j i}-\phi_{j}{ }^{h} \phi_{k i}\right)+c^{\prime} \phi_{i}{ }^{h} \phi_{k j}
$$

with absolute constants $c$ and $c^{\prime}$. If moreover the torsion tensor field has null covariant derivative, then the connection is the Levi-Civita's one and the manifold is a Kählerian manifold of constant holomorphic curvature.

\section{BIBLIOGRAPHY}

[1] T.FUKAMI, Invariant tensors under the real representation of unitary group and their applications, J. Math. Soc. Japan., 10 (1958), 135-144.

[2] N.H. KUIPER AND K. YANO, Two algebraic theorems with applications, Indag. Math., 18(1956), 319-328.

[3] M. OBATA, Affine connections on manifolds with almost complex, quateinion or Hermitian structure, Jap. J. Math., 26(1956), 43-77.

[4] K. YANO, On $n$-dimensional Riemannian spaces admitting a group of motions of order $n(n-1) / 2+1$, Trans. Amer. Math. Soc., 74(1953), 260-279.

DEPARTMENT OF MATHEMATICS, TOKYO METROPOLITAN UNIVERSITY. 